\title{
Grey-zone nel trattamento del paziente con Piede Diabetico. I risultati di una Delphi survey italiana multidisciplinare condivisa tra esperti
}

\author{
Roberto Anichini, ${ }^{1}$ Enrico Brocco, ${ }^{2}$ Marco Cavallini, ${ }^{3}$ Roberto Da Ros, ${ }^{4}$ Luigi Uccioli, ${ }^{5}$ Ciro Pempinello, ${ }^{6}$ \\ Elisabetta Salutini, ${ }^{1}$ Maurizio Volpini, ${ }^{7}$ Alice Volpini, ${ }^{7}$ Luca Monge ${ }^{8}$
}

${ }^{1}$ Unità Operativa Semplice Diabetologia, Diabetic Foot Unit USL Centro Toscana Area Pistoiese, Pistoia; ${ }^{2}$ Unità Operativa per il trattamento del piede diabetico Policlinico Abano Terme; ${ }^{3}$ Dipartimento di Scienze Medico Chirurgiche e di Medicina Traslazionale Azienda Ospedaliera Sant'Andrea, Roma; ${ }^{4}$ Centro Diabetologico Monfalcone (GO) -Ass2, Gorizia; ${ }^{5}$ Dipartimento di Me-dicina Interna, Unità di Piede Diabetico, Policlinico Universitario Tor Vergata, Roma; ${ }^{6}$ Dipartimento di Ortopedia e Traumatologia, Ospedale San Giovanni Bosco, Napoli; ${ }^{7}$ Libero professionista, Frascati (Roma); ${ }^{8}$ AOU Città della Salute e della Scienza di Torino; SSD Gestione Complicanze del Diabete e Piede Diabetico, Torino, Italia

\section{RIASSUNTO}

Il piede diabetico rappresenta una delle complicanze più severe del Diabete Mellito (DM) oltre che la prima causa di amputazione non traumatica degli arti inferiori; si stima, infatti, che ogni anno più di un milione di persone con DM siano sottoposte a trattamento demolitivo come conseguenza di questa condizione. Nonostante l'esistenza di linee guida nazionali ed internazionali sul management del piede diabetico, per alcuni aspetti gestionali le raccomandazioni proposte non raggiungono un forte livello di evidenza. Ciò rappresenta una potenziale fonte di incertezza decisionale, ma anche uno spunto da cui partire per chiarire aspetti legati al management del piede diabetico nella realtà italiana. L'obiettivo del presente documento è, quindi, quello di riadattare le raccomandazioni internazionali sulla gestione del piede diabetico alla luce dell'opinione di esperti italiani.

\section{INTRODUZIONE}

Ogni anno più di un milione di persone con Diabete Mellito (DM) sono sottoposte ad amputazione non traumatica degli arti inferiori come conseguenza di questa condizione. Diverse sono le aree di incertezza decisionale relative al management del piede diabetico. La presente rassegna raccoglie l'opinione di esperti italiani in merito

Corrispondenza: Roberto Anichini, Unità Operativa Semplice Diabetologia, DiabeticFoot Unit USLCentro Toscana Area Pistoiese, Pistoia 51100, Italy.

E-mail: roberto.anichini@uslcentro.toscana.it

Parole chiave: piede diabetico, diabete mellito, ulcera.

Finanziamenti: Il lavoro è stato realizzato con il contributo incondizionato di Angelini Pharma.

Conflitto d'interessi: Gli autori dichiarano l'assenza di conflitti di interesse.

Ricevuto per la pubblicazione: 29 dicembre 2019.

Accettato per la pubblicazione: 24 luglio 2020.

This work is licensed under a Creative Commons Attribution NonCommercial 4.0 License (CC BY-NC 4.0).

${ }^{\circ}$ Copyright: the Author(s), 2020

Licensee PAGEPress, Italy

Italian Journal of Wound Care 2020; 4(2):77-84

doi:10.4081/ijwc.2020.56 agli aspetti per cui le raccomandazioni delle linee guida sono caratterizzate da un basso livello di evidenza.

\section{DIABETE MELLITO: INQUADRAMENTO DELLA PATOLOGIA E COMPLICANZE AD ESSA ASSOCIATE}

Il DM è una patologia metabolica, caratterizzata da iperglicemia come conseguenza di una complessa interazione tra fattori genetici, immunologici ed ambientali. Alla base della patologia vi sono la perdita delle cellule beta pancreatiche e il deficit insulinico nel diabete tipo 1, l'insulinoresistenza, l'alterata secrezione insulinica e l'incrementata produzione epatica di glucosio nel diabete tipo 2 .

L'iperglicemia che caratterizza il diabete mellito provoca, nel tempo, l'insorgenza di complicanze micro- e macro-vascolari, spesso severe, che possono causare disabilità e che hanno un notevole impatto sul paziente $\mathrm{e}$ sul sistema sanitario. Tali complicanze vengono distinte in complicanze in microvascolari (retinopatia, nefropatia e neuropatia), macrovascolari (coronaropatia, arteriopatia obliterante periferica e malattia cerebrovascolare) e non vascolari (alterazioni cutanee ed infezioni). Le complicanze del diabete mellito sono la principale causa di morbilità e mortalità associate alla patologia, infatti, possono condurre allo sviluppo di insufficienza renale cronica, cecità, amputazioni non traumatiche agli arti inferiori, infarto e ictus. L'incidenza di DM è in aumento a livello mondiale con circa 250.000 nuovi caso/anno; questo incremento è riconducibile prevalentemente al- 
l'invecchiamento della popolazione e ad alcuni fattori di rischio (sovrappeso, obesità e sedentarietà). Secondo l'Organizzazione Mondiale della Sanità (OMS) la prevalenza di DM è pari a 52 milioni di persone affette in Europa e 346 milioni nel mondo, con una notevole variabilità in funzione dell'area geografica. Secondo i dati ISTAT 2015 in Italia il 5.4\% della popolazione è affetto da diabete: ${ }^{1,2}$ il $90 \%$ è rappresentato dal DM tipo 2 ed il $10 \%$ dal DM tipo 1. I dati dell'osservatorio Arno recentemente pubblicati indicano una prevalenza del diabete in Italia pari al $6.2 \% .^{3}$

\section{PIEDE DIABETICO: PREVALENZA, PATOGENESI E RELATIVE COMPLICANZE}

Il piede diabetico, una delle complicanze più severe del DM, è definito dalla presenza di ulcerazione e/o distruzione dei tessuti profondi associate ad un grado variabile di neuropatia ed arteriopatia periferiche. Il piede diabetico rappresenta la prima causa di amputazione non traumatica degli arti inferiori (che nell' $85 \%$ dei casi è preceduta da un'ulcerazione) nonché un frequente motivo di ricovero ospedaliero ${ }^{3,4}$ ed un fattore prognostico negativo di mortalità. In particolare, in uno studio multicentrico prospettico su 917 ulcerazioni del piede in pazienti diabetici la guarigione delle lesioni è risultata un fattore indipendente predittivo dell'aspettativa di vita e del rischio di amputazione. ${ }^{5}$ La prevalenza e l'incidenza delle ulcere nel piede diabetico sono variabili e spesso discordanti nei diversi studi riportati in letteratura. Complessivamente, si stima che in Europa la prevalenza di lesioni ulcerative al piede in pazienti diabetici sia pari al 5.5\%, due terzi di queste lesioni vanno incontro a guarigione mentre poco meno del $30 \%$ esita in amputazione. ${ }^{6}$ Si stima che globalmente, ogni 20 secondi, un paziente perda un arto a causa delle complicanze del diabete. ${ }^{4}$ I fattori di rischio per lo sviluppo di lesioni ulcerative al piede includono il sesso maschile, il tabagismo, il DM tipo 2, l'età avanzata, la durata di malattia superiore a $10 \mathrm{anni}$, la neuropatia periferica e/o l'arteriopatia obliterante a carico degli arti inferiori, l'ipertensione, la retinopatia diabetica, la nefropatia diabetica, lo scarso controllo glicemico, un'anamnesi positiva per precedenti ulcerazioni, la presenza di alterazioni strutturali del piede (presenza di alterazioni ossee, callosità, ispessimenti ungueali), l'uso di calzature inappropriate. La patogenesi delle complicanze a carico degli arti inferiori nei pazienti diabetici è multifattoriale ed in essa hanno un ruolo diversi fattori tra cui la neuropatia e vasculopatia periferica sono fondamentali. La neuropatia periferica, interferendo con la nocicezione, determina ipoestesia e quindi predispone a traumi inavvertiti perché non associati a dolore. Inoltre, l'alterazione della capacità propriocettiva, causando un'anomalia di carico in fase di deambulazione, determina stress biomec- canico, sofferenza a livello dermico con conseguente ipossia cellulare, emorragie sottocutanee ed ulcerazioni tissutali; infine, la neuropatia autonomica, che si manifesta con anidrosi ed alterazioni del microcircolo predispone a secchezza cutanea e fissurazioni. L'arteriopatia periferica è presente nel $50 \%$ dei pazienti con ulcera di piede diabetico ed è generalmente causata da aterosclerosi con diminuzione od interruzione del flusso ematico in una o più arterie dell'arto inferiore. ${ }^{7}$ Tuttavia, solo una piccola percentuale di tali ulcerazioni è puramente ischemica, essendo la maggior parte di esse neuropatiche o neuro-ischemiche (ovvero causate dalla simultanea presenza di neuropatia ed ischemia). ${ }^{8}$ Le ulcerazioni neuropatiche si localizzano tipicamente in zone di ipercarico come le teste metatarsali, hanno contorno irregolare con margini perilesionali ipercheratosici e sono caratterizzate da assenza di dolore. Le lesioni ischemiche sono tipicamente localizzate sul tallone, apice e dorso delle dita, spazi interdigitali, hanno margini regolari, ben definiti con cute perilesionale atrofica, inoltre il più delle volte sono accompagnate da dolore intenso. ${ }^{8}$

Un rapido inquadramento nosologico dell'ulcera è indispensabile per un'adeguata gestione delle lesioni ulcerative e delle complicanze ad esse associate. Inoltre l'eventuale presenza infezione dell'ulcera è associata ad un notevolmente aumento del rischio amputativo. La diagnosi clinica di infezione può essere posta in presenza di almeno 2 classici segni di infiammazione locale tra cui rossore, gonfiore, dolore, indurimento/edema, calore al termo tatto o essudato purulento. Nei casi più gravi l'infezione e l'essudato possono andare a coinvolgere i tessuti molli più profondi estendendosi lungo le guaine tendinee fino allo sviluppo di un flemmone con interessamento degli spazi sotto fasciali del dorso o della pianta del piede.

\section{APPROFONDIMENTO DELLE CRITICITÀ/GREYZONES EMERSE DALLA CONSENSUSDELPHI}

Il metodo Delphi è un metodo d'indagine interattivo che consente un confronto tra un panel di esperti con l'obiettivo primario di far convergere l'opinione di ciascun membro in una posizione più completa e condivisa (consensus). I punti per i quali non si raggiunge una posizione di consenso rispecchiano la complessità del DM soprattutto in relazione agli aspetti di gestione e trattamento multidisciplinare del piede diabetico. Le criticità emerse, definite con il termine di grey zones, corrispondono a quei temi per i quali i dati in letteratura risultano insufficienti e dove le Linee Guida non appaiono dirimenti. L'indagine (survey) che è stata condotta in precedenza si è posta l'obiettivo di esplorare il punto di vista di un panel di esperti italiani sul management del piede diabetico. ${ }^{9}$ 


\section{RISULTATI}

I risultati della survey evidenziano criticità riconducibili a 7 items di non consenso. Verranno proposte le tabelle di non consenso 1-3-4-6-8, per le altre si rimanda ai risultati della survey precedentemente pubblicata. ${ }^{9}$

Il primo elemento di non consenso, seppur con un trend verso l'accordo tra gli esperti, riguarda la fisiopatologia delle lesioni del piede diabetico (item 1.4, Tabella 1). In particolare, non c'è pieno accordo nel ritenere che le lesioni di piede diabetico siano prevalentemente determinate dalla neuropatica periferica. Ciò può essere ascrivibile all'estrema complessità patogenetica della sindrome del piede diabetico; infatti, sebbene nella componente neuropatica rivesta un ruolo fondamentale, l'aspetto vascolare viene spesso percepito dai clinici come motivo primario per cui i pazienti ischemici vengono considerati ad uno stadio di malattia più avanzato rispetto ai soggetti puramente neuropatici. Inoltre, almeno in parte, l'impostazione formale dello statement potrebbe aver rappresentato un elemento foriero di non consenso; in particolare, la dizione "impairment macro-vascolare" sembra includere un concetto più ampio, passibile di interpretazioni differenti. Tra queste emerge il punto di vista del chirurgo vascolare per il quale l'arteriopatia periferica distale, tipica del paziente diabetico determina un coinvolgimento della circolazione collaterale e delle piccole arterie delle arcate vascolari del piede condizionando il letto di accoglimento (run-off) per le procedure di rivascolarizzazione. Comunque, dalla revisione delle più recenti Linee Guida sul tema, ${ }^{8}$ viene sottolineato come la maggior parte delle ulcerazioni sia prevalentemente neuro-ischemica, sottolineando quindi l'importanza eziopatogenetica della neuropatia (Tabella 1).

Il secondo elemento di non consenso riguarda due items relativi al ruolo dell'antisettico (Tabella 2).

Il primo (item 3.5) documenta il non accordo nel ritenere che sia fondamentale applicare un antisettico per prevenire l'infezione di piede diabetico, al fine di garantirne la guarigione in tempi fisiologici. Il panel di esperti ritiene che una possibile spiegazione del mancato raggiungimento di consenso dipenda da una non chiara formulazione del quesito, responsabile quindi di una non univoca interpretazione del concetto "tempi fisiologici". Il ruolo dell'antisettico dipende dal tipo di lesione: in caso di lesione superficiale è sufficiente un'irrigazione con soluzione fisiologica, viceversa nel caso di una lesione profonda, di un flemmone o in presenza di evidenti segni di infezione, è raccomandato l'impiego di un antisettico al fine di prevenire l'estensione del processo infettivo ai tessuti profondi, poiché questo esporrebbe il paziente ad un aumentato rischio di osteomielite ed, in casi estremi, alla necessità di procedere con un intervento di amputazione minore o maggiore. In merito a questa tematica, è emerso inoltre che nei casi di lesioni ricoperte da biofilm è necessaria un'accurata disinfezione prima di qualsivoglia terapia. È risultato altresì condivisibile l'impiego di un antisettico nelle lesioni complesse ${ }^{10}$ al fine di consentire la rimozione dell'essudato e la riduzione della carica batterica, accelerando così i tempi di guarigione che tendono, invece, a rallentare in presenza di infezione locale conclamata. Inoltre, l'uso di un antisettico appare cruciale in due contesti specifici: in occasione delle prime medicazioni in fase di presa in carico del paziente (fase in cui

Tabella 1. Definizioni e classificazioni - Ritengo che:

\begin{tabular}{|c|c|c|c|c|c|c|}
\hline$\Delta$ & 1 & 2 & 3 & 4 & 5 & ТОT \\
\hline \multirow[t]{2}{*}{$\begin{array}{l}\text { 1.1. le lesioni del piede diabetico siano l'espressione di una manifestazione } \\
\text { locale piutt osto chel'evoluzione di una sindrome generale }\end{array}$} & 23 & 7 & 1 & 1 & 0 & 32 \\
\hline & \multicolumn{2}{|c|}{$94 \%$} & \multicolumn{3}{|c|}{$6 \%$} & $100 \%$ \\
\hline \multirow[t]{2}{*}{$\begin{array}{l}1.2 \text { le classificazioni delle lesioni attualmente utilizzate siano necessarie per } \\
\text { la corretta gestione delle lesioni del piede diabetico }\end{array}$} & 0 & 0 & 5 & 17 & 10 & 32 \\
\hline & \multicolumn{2}{|c|}{$0 \%$} & \multicolumn{3}{|c|}{$100 \%$} & $100 \%$ \\
\hline \multirow{2}{*}{$\begin{array}{l}\text { 1.3. sia utile classificare le infezioni del piede diabetico in lievi, moderate } \\
\text { (dass Ae B) e gravi ai fini di un corretto management diagnostico } \\
\text { terapeutico, secondo IDSA2012 }\end{array}$} & 0 & 2 & 4 & 13 & 13 & 32 \\
\hline & \multicolumn{2}{|c|}{$6 \%$} & \multicolumn{3}{|c|}{$94 \%$} & $100 \%$ \\
\hline \multirow[t]{2}{*}{$\begin{array}{l}\text { 1.4. che la fisiopatogenes di una lesione di piede diabetico sa } \\
\text { prevalentemente sostenuta da un substrato di neuropatia periferica rispetto } \\
\text { ad un impairment macrovascolare }\end{array}$} & 4 & 8 & 10 & 9 & 1 & 32 \\
\hline & \multicolumn{2}{|c|}{$36 \%$} & \multicolumn{3}{|c|}{$64 \%$} & $100 \%$ \\
\hline
\end{tabular}


è maggiore il rischio di avere a che fare con un'infezione della lesione, fino ad allora verosimilmente mai gestita) ed in caso di interventi con innesti cutanei (fase in cui la presenza di infezione potrebbe esitare in un fallimento dell'intervento di chirurgia plastica per mancato attecchimento dell'innesto). Recentemente nei casi dubbi grazie alla diagnostica mediante fluorescenza esiste la possibilità di evidenziare le ferite con una carica batterica $>10^{4} \mathrm{CFU}$. $\mathrm{Al}$ contrario, l'uso dell'antisettico non è raccomandato nelle fasi in cui il fondo della lesione appare dominato dalla produzione di tessuto di granulazione attivo; in questi casi è infatti preferibile una semplice detersione con cristalloidi e/o soluzione fisiologica. È documentato come la coesistenza di uno stato infettivo e dell'insufficienza arteriosa dell'arto inferiore triplichino il rischio di non guarigione di una ulcera rispetto ai casi con neuropatia o vasculopatia ma senza infezione..$^{11} \mathrm{E}$, quindi, fondamentale tipizzare il paziente e il tipo di lesione, al fine di personalizzare i tempi di intervento, le figure professionali coinvolte e il trattamento più adeguato.

L'item 3.6 documenta un secondo elemento di non consenso relativo alla convinzione che sia fondamentale applicare un antisettico per facilitare la guarigione in tempi fisiologici (il trend della risposta tende verso il consenso positivo). Anche per questo item il panel di esperti ritiene che la formulazione del quesito renda possibili diverse interpretazioni nonostante avesse il solo scopo di "provocare" ciascun clinico a descrivere il proprio approccio quotidiano, focalizzandosi su esperienza perso- nale e strumenti a disposizione, specialmente nei casi in cui le Linee Guida risultano difficilmente applicabili. Considerando la dizione "tempi fisiologici" quale elemento di non consenso, si è optato per una sua interpretazione come sinonimo di "tempi attesi".

Per quanto concerne gli elementi critici relativi alla fase di presa in carico e monitoraggio nel tempo di un paziente affetto da lesioni correlate al piede diabetico, l'item 4.8 (Tabella 3) documenta il non consenso nel ritenere che sia indispensabile la valutazione degli indici di flogosi per l'inquadramento dell'infezione di piede diabetico. Il panel di esperti concorda nel considerare tra le possibili cause di non consenso l'interpretazione soggettiva del termine "inquadramento" da non confondere con il concetto di "diagnosi". Esistono infatti criteri validati per la diagnosi di infezione di lesioni del piede diabetico. In particolare, gli stati di iperglicemia, apparentemente ingiustificati ma in realtà legati ad uno stato infiammatorio, e le alterazioni degli indici di flogosi (proteina $\mathrm{C}$ reattiva e procalcitonina) sono elementi cruciali per valutare l'effetto della terapia, la necessità di una modifica dell'iter terapeutico e il follow up mentre il loro ruolo in fase diagnostica è ritenuto marginale.

L'item 6.4 (Tabella 4) descrive il non consenso nel ritenere che nel wound care la scelta della medicazione avanzata sia determinante per la guarigione della ferita, sollevando perplessità interpretative da parte del panel di esperti secondo cui, il ragionamento clinico avrebbe dovuto portare all'ottenimento di un consenso positivo poi-

Tabella 2. Ruolo dell'antisettico - Ritengo che:

\begin{tabular}{|c|c|c|c|c|c|c|}
\hline 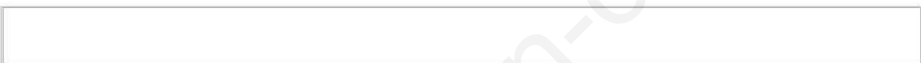 & 1 & 2 & 3 & 4 & 5 & ТОI \\
\hline \multirow[t]{2}{*}{$\begin{array}{l}\text { 3.1. sia fondamentale un iniziale momento di disinfezione delle lesioni di piede } \\
\text { diabetion, prima di qualsiasi approccio diagnostio-ter apeutico al fine di garantirne } \\
\text { il sucossivo management ottimale della lesione }\end{array}$} & 0 & 6 & 6 & 10 & 10 & 32 \\
\hline & \multicolumn{2}{|c|}{$18 \%$} & \multicolumn{3}{|c|}{$82 \%$} & $100 \%$ \\
\hline \multirow[t]{2}{*}{$\begin{array}{l}\text { 3.2. le car atteristiche ideali di un antisettico da utilizzare in questo setting siano la } \\
\text { rapidità d'azione e l'ampio spettro. }\end{array}$} & 0 & 1 & 5 & 17 & 9 & 32 \\
\hline & \multicolumn{2}{|c|}{$3 \%$} & \multicolumn{3}{|c|}{$97 \%$} & $100 \%$ \\
\hline \multirow[t]{2}{*}{$\begin{array}{l}3.3 \text { la citotossicità sia un criterio preso in consider azione per la scelta } \\
\text { dell'antisettico }\end{array}$} & 0 & 1 & 9 & 13 & 9 & 32 \\
\hline & \multicolumn{2}{|c|}{$3 \%$} & \multicolumn{3}{|c|}{$97 \%$} & $100 \%$ \\
\hline \multirow[t]{2}{*}{ 3.4. I'assenza di œolorazione sia una caratteristica fondamentale di un antisettic } & 0 & 5 & 9 & 7 & 11 & 32 \\
\hline & \multicolumn{2}{|c|}{$15 \%$} & \multicolumn{3}{|c|}{$85 \%$} & $100 \%$ \\
\hline \multirow[t]{2}{*}{$\begin{array}{l}\text { 3.5. sia fondamentale applicare un antisettico per prevenire l'infezione di piede } \\
\text { diabetio al fine di garantirne la guarigione in tempi fisiologid }\end{array}$} & 1 & 14 & 10 & 4 & 3 & 32 \\
\hline & \multicolumn{2}{|c|}{$48 \%$} & \multicolumn{3}{|c|}{$52 \%$} & $100 \%$ \\
\hline \multirow[t]{2}{*}{$\begin{array}{l}\text { 3.6. sia fondamentale applicare un antisettio al fine di facilitare la guarigione in } \\
\text { tempi fisiologid }\end{array}$} & 1 & 11 & 10 & 8 & 2 & 32 \\
\hline & \multicolumn{2}{|c|}{$36 \%$} & \multicolumn{3}{|c|}{$64 \%$} & $100 \%$ \\
\hline
\end{tabular}


ché le medicazioni avanzate sono differenziate e hanno indicazione d'impiego in relazione alle peculiari fasi del processo di guarigione, inserendosi come ausili di una corretta wound bed preparation secondo il concetto sintetizzato nell'acronimo TIME (gestione corretta del tessuto, trattamento dell'infezione, mantenimento di un ambiente umido e facilitazione della rigenerazione del margine epiteliale della lesione). Inoltre i cinque approcci terapeutici (standard wound care, farmaci biologici, fattori di crescita piastrinici, terapia cellulare e terapia iper- barica) presi in considerazione non sono applicati in modo uniforme dai clinici del panel. Questa scarsa condivisione di approccio potrebbe derivare dal fatto che esistono scarse evidenze scientifiche in merito al ruolo di ciascuno di questi interventi. Il board concorda nel ritenere che una medicazione avanzata acceleri il processo di guarigione creando un microambiente umido, adeguato e più fisiologico rispetto a quanto si otterrebbe con delle medicazioni tradizionali. Vista la carenza di Linee Guida basate su evidenze scientifiche autorevoli riguardo all'impiego di me-

Tabella 3. Diagnosi - Ritengo che:

\begin{tabular}{|c|c|c|c|c|c|c|}
\hline & 1 & 2 & 3 & 4 & 5 & TOT \\
\hline \multirow[t]{2}{*}{$\begin{array}{l}4.6 \text { sia utile effettuare un tampone superficiale dell'ulcera } \\
\text { attraver so rolling superficiale }\end{array}$} & 11 & 15 & 4 & 2 & 0 & 32 \\
\hline & \multicolumn{2}{|c|}{$82 \%$} & \multicolumn{3}{|c|}{$18 \%$} & $100 \%$ \\
\hline \multirow[t]{2}{*}{$\begin{array}{l}4.7 \text { sia utile l'impiego di metodiche alter native come l'aspirazione } \\
\text { mediate ago delle secrezioni di ferita }\end{array}$} & 0 & 9 & 14 & 2 & 7 & 32 \\
\hline & \multicolumn{2}{|c|}{$27 \%$} & \multicolumn{3}{|c|}{$73 \%$} & $100 \%$ \\
\hline \multirow[t]{2}{*}{$\begin{array}{l}4.8 \text { sia indispensabile la valutazione degli indici di flogosi per } \\
\text { l'inquadramento dell'infezione di piede diabetico }\end{array}$} & 2 & 11 & 10 & 3 & 6 & 32 \\
\hline & \multicolumn{2}{|c|}{$39 \%$} & \multicolumn{3}{|c|}{$61 \%$} & $100 \%$ \\
\hline \multirow[t]{2}{*}{$\begin{array}{l}4.9 \text { sia necessario eseguire delle emocolture in presenza di ulcera } \\
\text { infetta di piede diabetico }\end{array}$} & 4 & 19 & 5 & 3 & 1 & 32 \\
\hline & \multicolumn{2}{|c|}{$73 \%$} & \multicolumn{3}{|c|}{$27 \%$} & $100 \%$ \\
\hline
\end{tabular}

Tabella 4. Altri approcci terapeutici - Ritengo che:

\begin{tabular}{|c|c|c|c|c|c|c|}
\hline$N$ & 1 & 2 & 3 & 4 & 5 & TOT \\
\hline \multirow[t]{2}{*}{$\begin{array}{l}\text { 6.1. dopo } 4 \text { settimane di "standard wound care" senza aver } \\
\text { ottenuto una riduzione di almeno del } 50 \% \text { vada riconsiderata la } \\
\text { stadiazione della lesione }\end{array}$} & 0 & 0 & 8 & 16 & 8 & 32 \\
\hline & \multicolumn{2}{|c|}{$0 \%$} & \multicolumn{3}{|c|}{$100 \%$} & $100 \%$ \\
\hline \multirow{2}{*}{$\begin{array}{l}6.2 \text { in caso di mancato miglioramento della lesione sia necessario } \\
\text { utilizzare farmaci biologici, fattori di crescita piastrinici, terapia } \\
\text { cellulare e terapia iperbarica. }\end{array}$} & 3 & 18 & 9 & 2 & 0 & 32 \\
\hline & \multicolumn{2}{|c|}{$67 \%$} & \multicolumn{3}{|c|}{$33 \%$} & $100 \%$ \\
\hline \multirow[t]{2}{*}{$\begin{array}{l}6.3 \text { in caso di mancato miglior amento della lesione è } \\
\text { indispensabile la rivalutazione vascolare. }\end{array}$} & 0 & 0 & 3 & 14 & 15 & 32 \\
\hline & \multicolumn{2}{|c|}{$0 \%$} & \multicolumn{3}{|c|}{$100 \%$} & $100 \%$ \\
\hline \multirow[t]{2}{*}{$\begin{array}{l}6.4 \text { nel wound care la scelta della medicazione avanzata sia } \\
\text { determinante per la guarigione della ferita }\end{array}$} & 1 & 15 & 9 & 3 & 4 & 32 \\
\hline & \multicolumn{2}{|c|}{$48 \%$} & \multicolumn{3}{|c|}{$52 \%$} & $100 \%$ \\
\hline \multirow[t]{2}{*}{$\begin{array}{l}6.5 \text { la presenza di un'infezione grave richieda l'ospedalizzazione al } \\
\text { fin di garantire la corretta gestione in termini diagnostici e } \\
\text { terapeutici }\end{array}$} & 0 & 1 & 2 & 10 & 19 & 32 \\
\hline & \multicolumn{2}{|c|}{$3 \%$} & \multicolumn{3}{|c|}{$97 \%$} & $100 \%$ \\
\hline
\end{tabular}


dicazioni avanzate con prodotti ad azione antibatterica, il board ritiene che l'utilizzo di queste medicazioni, in particolare la terapia a pressione negativa (negative pressure wound therapy NPWT) e le medicazioni a base di sucralfato, debba basarsi principalmente sull'esperienza e sul giudizio clinico. Le più recenti Linee Guida dell'IWGDF ${ }^{4}$ hanno infatti confermato che la NPWT trova indicazione come trattamento adiuvante alla Best Standard Of Care (BSC) per ridurre le dimensioni delle lesioni in fase postchirurgica ma suggeriscono di non usarla in sostituzione della BSC nelle ferite non chirurgiche. Le stesse linee guida, con raccomandazione dalla forza DEBOLE e con Qualità delle evidenze MODERATA, invitano a considerare l'utilizzo di medicazioni a base di sucralfato nelle ulcere neuroischemiche non infette che non abbiano risposto ai trattamenti BSC.

Nello statement viene inoltre specificato che, nel processo di guarigione, non è determinante solo la medicazione applicata ma anche l'adeguata gestione del letto della ferita TIME e del paziente nel suo complesso, in una presa in carico olistica. ${ }^{12}$

Lo statement 8 relativo alla chirurgia presenta due elementi di non consenso (Tabella 5).

L'item 8.3 descrive il non consenso nel ritenere che l'amputazione maggiore primaria sia una valida opzione chirurgica in caso di segni sistemici di infezione, tuttavia il termine "opzione" non sembra adeguato ed il board concorda sul fatto che l'amputazione primaria non rappresenta "un'opzione" ma va considerata come estrema ratio nei casi di infezione sistemica con manifestazioni cliniche tipiche tra cui febbre, dolore intenso, malessere generale, squilibrio metabolico e compromissione d'or- gano (singoli segni di infezione, come la presenza di iperpiressia persistente isolata non rappresentano, a parere del panel, un'indicazione all'amputazione). In caso di lesione infetta, gli esperti concordano nel ritenere il management conservativo (preparazione del letto della ferita, debridement accurato e gestione dei segni sistemici) l'approccio di prima scelta. Di estrema rilevanza appare la presenza di un team multidisciplinare che gestisca adeguatamente un trattamento conservativo tempestivo e prolungato.

L'item 8.4 sottolinea invece il non consenso (seppur con un trend verso il consenso negativo) nel ritenere che l'amputazione maggiore debba essere preferita ad un trattamento conservativo ripetuto e prolungato di una lesione grave. La corretta gestione di una lesione grave è condizionata da due criticità: da un lato $i$ costi sanitari ${ }^{13}$ correlati al trattamento conservativo e che spesso non considerano $i$ costi diretti della protesizzazione e riabilitazione del paziente ed indiretti a carico del nucleo familiare, e dall'altro $i$ dati presenti in letteratura che evidenziano un aumentato tasso di mortalità tra i pazienti sottoposti ad amputazione maggiore. Nonostante la disomogeneità di approcci e risorse disponibili sul territorio, il panel di esperti sottolinea la necessità di attenersi alle Linee Guida, che rappresentano uno strumento di buona pratica clinica, oltre che di tutela per il clinico chiamato a gestire situazioni complesse non scevre di risvolti medico-legali.

\section{PARERE DEGLI ESPERTI NELLA GESTIONE DEL PIEDE DIABETICO}

I fattori che portano allo sviluppo di ulcere al piede nei pazienti diabetici sono complessi e richiedono l'inter-

Tabella 5. Chirurgia - Ritengo che:

\begin{tabular}{|c|c|c|c|c|c|c|}
\hline$x_{n}$ & 1 & 2 & 3 & 4 & 5 & TOTAL \\
\hline \multirow{2}{*}{$\begin{array}{l}8.1 \text { the presence of gases in the tissues surrounding the ulcer with } \\
\text { the presence of necrotised fasciitis is an appropriate indication for } \\
\text { urgent surgery }\end{array}$} & 0 & 0 & 2 & 4 & 26 & 32 \\
\hline & \multicolumn{2}{|c|}{$0 \%$} & \multicolumn{3}{|c|}{$100 \%$} & $100 \%$ \\
\hline \multirow{2}{*}{$\begin{array}{l}8.2 \text { In the presence of severe infected lesions (cellulitis, fasciitis, } \\
\text { phlegmon or abscess) urgent surgery is warranted }\end{array}$} & 0 & 0 & 2 & 3 & 27 & 32 \\
\hline & \multicolumn{2}{|c|}{$0 \%$} & \multicolumn{3}{|c|}{$100 \%$} & $100 \%$ \\
\hline \multirow{2}{*}{$\begin{array}{l}8.3 \text { major primary amputation is a valid surgical option in case of } \\
\text { systemic signs of infection }\end{array}$} & 6 & 13 & 4 & 5 & 4 & 32 \\
\hline & \multicolumn{2}{|c|}{$61 \%$} & \multicolumn{3}{|c|}{$39 \%$} & $100 \%$ \\
\hline \multirow[t]{2}{*}{$\begin{array}{l}8.4 \text { major amputation should be preferred to repeated and } \\
\text { prolonged conservative treatment of a severe peripheral lesion }\end{array}$} & 5 & 14 & 3 & 5 & 5 & 32 \\
\hline & \multicolumn{2}{|c|}{$61 \%$} & \multicolumn{3}{|c|}{$39 \%$} & $100 \%$ \\
\hline
\end{tabular}


vento di più figure professionali, con molteplici trattamenti da realizzare nell'ambito di un periodo di assistenza prolungato. Gli operatori sanitari esperti in differenti specialità dovrebbero, quindi, essere coinvolti nella gestione del piede diabetico, al fine di dare un contributo specifico in relazione al proprio settore di esperienza. Tra i professionisti sanitari coinvolti nella cura del piede diabetico il podologo svolge un ruolo fondamentale; tale operatore infatti interviene in fase di presa in carico del paziente quando è maggiore il rischio di infezione della lesione, mettendo in atto le prime medicazioni. In una fase più avanzata, la presenza di infezione in pazienti sottoposti ad interventi che prevedano l'impiego di innesti cutanei potrebbe esitare in un mancato attecchimento dell'innesto stesso, costituendo quindi controindicazione all'esecuzione di questa procedura chirurgica, e richiedendo ulteriore azione clinica per la sua eliminazione. Dal punto di vista del chirurgo vascolare, considerato che le procedure di rivascolarizzazione, in questi pazienti, spesso consentono una pervietà primaria solo di alcuni mesi, è opportuno considerare il corretto timing per l'intervento di rivascolarizzazione. Sebbene le procedure di rivascolarizzazione siano efficaci nel trattamento della condizione di insufficienza vascolare/ischemia, il loro impiego esteso è probabilmente sopravvalutato se considerato rispetto ai miglioramenti ottenuti con la corretta e meno invasiva gestione della lesione soprattutto in caso del piede neuroischemico.

\section{MANAGEMENT DEL PIEDE DIABETICO: NECESSITÀ DI UNA GESTIONE MULTIDISCIPLINARE}

Il management del piede diabetico ha come obiettivo primario la prevenzione di ulcere ed amputazioni (secondarie alla presenza di un'ulcera nell' $85 \%$ dei casi) al fine di ridurre l'inevitabile impatto economico e psicosociale. Data la complessità ed eterogeneità dei quadri clinici, è necessaria una gestione multidisciplinare che garantisca un'efficace collaborazione tra le figure professionali coinvolte. . $^{14,15}$

La recente pubblicazione del Position Statement del gruppo di Studio Italiano sul piede diabetico ha cercato di individuare i compiti specifici e le responsabilità dei singoli componenti del Team, indicando al tempo stesso chi fa cosa, nella presa in carico del paziente con piede diabetico. ${ }^{16}$

A questo si aggiunge la necessità di coinvolgere ed educare il paziente all'autogestione attraverso la cura del piede e l'uso di calzature adeguate. Il modello assistenziale auspicabile dovrà, quindi, prevedere la cooperazione tra diversi professionisti (medico di medicina generale, diabetologo, neurologo, ortopedico, chirurgo generale, chirurgo vascolare, infermiere, dietista, podologo, tecnico ortopedico, psicologo e/o psichiatra) che operino al fine di garantire la presa in carico del paziente in una visione olistica, con modalità comunicative e relazionali adeguate. ${ }^{17}$ Le evidenze disponibili, a partire da una serie di studi osservazionali italiani, mostrano che la partecipazione attiva dei centri diabetologici, accanto alla medicina generale, sia associata ad una consistente riduzione della mortalita. ${ }^{18-22}$ Infine, la presenza di un team multidisciplinare per la cura del piede diabetico ha dimostrato una riduzione nel tasso di amputazione compresa tra il $49 \mathrm{e}$ $1^{\prime} 85 \%{ }^{17}$ Per gli operatori del team si rende pertanto necessaria, attraverso l'implementazione di master universitari interdisciplinari dedicati, una formazione comune di base e di approccio al paziente ed alla lesione e la consapevolezza della necessità di un tempestivo invio in consulenza ai differenti specialisti del settore.

\section{L'ANTISETTICO "IDEALE": RUOLO NEL MANAGEMENT DEL PIEDE DIABETICO}

In relazione all'eziologia polimicrobica delle infezioni del piede diabetico il ruolo degli antisettici risulta essere cruciale. Una percentuale variabile dal $40 \%$ al $60 \%$ dei pazienti affetti da DM trattati per un'ulcera al piede riceve una terapia antibiotica. Sebbene l'uso dell'antisettico sia raccomandato per il trattamento delle ulcere infette, un uso incongruo può associarsi frequentemente a effetti avversi locali e dal manifestarsi di fenomeni di resistenza batterica. Pertanto le caratteristiche imprescindibili dell'antisettico "ideale" includono un'azione rapida e ad ampio spettro, la mancata interferenza con il processo di guarigione, non provocare irritazione locale, essere incolore permettendo di monitorare nel tempo il letto di ferita e non indurre resistenze batteriche nei ceppi trattati.

\section{LINEE GUIDA: STRUMENTO DI SUPPORTO O DA APPROFONDIRE}

Nonostante la varietà di approcci clinici e di risorse disponibili sul territorio italiano, le Linee Guida rappresentano sia uno strumento di buona pratica clinica sia un mezzo di tutela per il clinico chiamato a gestire situazioni complesse che spesso hanno anche risvolti medicolegali. Tuttavia, nonostante il management del piede diabetico sia oggetto di specifiche raccomandazioni, restano aperte questioni di gestione delle lesioni per le quali le linee di indirizzo non raggiungono un forte livello di evidenza o le singole esperienze sono particolarmente disomogenee. Un potenziale obiettivo futuro è l'organizzazione di trial internazionali che si focalizzino sulle criticità per le quali, al momento, i dati in Letteratura risultano insufficienti. 


\section{BIBLIOGRAFIA}

1. ISTAT. Rapporto annuale 2015. Disponibile presso: https:// www.istat.it/it/files/2015/05/Rapporto-Annuale-2015.pdf.

2. Gnavi R, Karaghiosoff L, Balzi D. Diabetes prevalence estimated using a standard algorithm based on electronic health data in various areas of Italy. EpidemiolPrev 2008;32:15-21.

3. Osservatorio Arno Diabete 2019. Il profilo assistenziale della popolazione con dia-bete. Disponibile presso: https://www.siditalia.it/clinica/linee-guida-societari/send/80-linee-guida-documenti-societari/5025-rapporto-arno-diabete-2019

4. IWGDF. The 2019 IWGDF Guidelines. Disponibile presso: https://iwgdfguidelines.org/wp-content/uploads/2019/05/ IWGDF-Guidelines-2019.pdf.

5. Taylor SM, Johnson BL, Samies NL, et al. Contemporary management of diabetic neuropathic foot ulceration: a study of 917 consecutively treated limbs. J Am Coll Surg 2011;212: 532-45.

6. Zhang P, Lu J, Jing Y, et al. Global epidemiology of diabetic foot ulceration: a sy-stematic review and meta-analysis. Ann Med 2017;49:106-16.

7. American Diabetes Association. Peripheral arterial disease in people with diabetes. Diabetes Care 2003;26:3333-41.

8 . Boulton AJM. Clinical presentation and management of diabetic neuropathy and foot ulceration. Diabet Med 1991; 8:552-7.

9. Salutini E, Brocco E, Da Ros R, et al. The complexity of diabetic foot management: from common care to best practice. The Italian expert opinion by Delphi survey. Int J Lower Extremity Wounds 2019;19:34-43.

10. European Pressure Ulcer Advisory Panel (EPUAP), National Pressure Ulcer Advisory Panel (NPUAP), Pan Pacific Pressure Injury Alliance (PPPIA). Prevention and treat-ment of pressure ulcers: clinical practice guidelines. Disponibile presso: https://cdn.ymaws.com/npiap.com/resource/resmgr/ 2014 guideline.pdf

11. Prompers L, Schaper N, Apelqvist J, et al. Prediction of outcome in individuals with diabetic foot ulcers: focus on the differences between individuals with and without peripheral arterial disease- The EURODIALE Study. Diabetologia 2008;51:747-55.

12. Schultz GS, Barillo DJ, Mozingo DW, et al. Wound bed pre- paration and a brief history of TIME. Wound Bed Advisory Board Members. Int Wound J 2004;1:19-32. doi: 10.1111/j.1742-481x.2004.00008.x.

13. International Working Group on the Diabetic Foot, Gruppo di Studio Intersocietario PiedeDiabetico SID-AMD. International Consensus on the Diabetic Foot \& Practical Guidelines on the management and prevention of the diabetic foot. 2016 (traduzio-ne italiana).

14. Dargis V, Pantelejeva O, Jonushaite A, et al. Benefits of a multidisciplinary ap-proach in the management of recurrent diabetic foot ulceration in Lithuania: a pro-spective study. Diabetes Care 1999;22:1428-31.

15. Hamonet J, Verdie-Kessler C, Daviet JC, et al. Evaluation of a multidisciplinary con-sultation of diabetic foot. Ann Phys Rehabil Med 2010;53:306-18

16. Anichini R, Brocco E, Caravaggi CM, et al.On behalf of SID/AMD Diabetic Foot Study Group. Physician experts in diabetes are natural team leaders for managing diabet-ic patients with foot complications. A position statement from the Italian diabetic foot study group. Nutr Metab Cardiovasc Dis2020;30:167-178. doi: 10.1016/j.numecd.2019.11.009.

17. Giusti A, Gawronski O, Maggini M. Istituto Superiore di Sanità. Gestione integrata del diabete: indagine qualitativa sulla percezione dell'adeguatezza e sui bisogni in-formativi. 2009. Disponibile presso: http://old.iss.it/binary/publ/cont/0935 WEB.pdf.

18. Bonora E, Monami M, Bruno G, et al. Attending Diabetes Clinics is associated with a lower all-cause mortality. A metaanalysis of observational studies performed in Italy. NutrMetabCardiovasc Dis 2018;28:431-5.

19. Zoppini G, Verlato G, Bonora E, Muggeo M. Attending the diabetes center is associ-ated with reduced cardiovascular mortality in type 2 diabetic patients: the Verona Diabetes Study. Diabetes Metab Res Rev 1999;15:170-4.

20. Pimouguet C, Le Goff M, Thiébaut R, et al. Effectiveness of disease-management programs for improving diabetes care: a meta-analysis. CMAJ 2011;183:115-27.

21. Knight K, Badamgarav E, Henning JM, et al. A systematic review of diabetes dis-ease management programs. Am J Manag Care 2005;11:242-50.

22. Norris SL, Nichols PJ, Caspersen CJ, et al. The effectiveness of disease and case management for people with diabetes. Am J Prev Med 2002;22:15-38. 